\title{
Blockchain: Taking the Genius of Financial Tracking into the Electronic Health Record Environment on a Global Basis
}

Ted Braid

Abstract: Blockchain technology developed in 2008 as part of a proposal for the Bitcoin virtual currency system-eschewing a central authority for issuing currency, transferring ownership, and confirming transactions. ${ }^{1}$ Before understanding its value for the goal of accurate, auditable, and easy of accessing medical record data on a global basis while still maintaining the security desired and mandated by the Health Insurance Portability and Accountability Act of 1996 (HIPAA), you must understand how it works.

\section{Blockchain as Designed for Financial Transactions}

A block is the "current" part of a blockchain, which records some or all recent transactions. Once completed, the transaction enters the blockchain as permanent data in the database. Each time a block is completed, a new block is generated. There are countless such blocks in the blockchain.

The blocks in a blockchain are linked to each other (like a chain) in proper linear, chronological order, with every block containing a hash of the previous block. The hash function allows you to store and find data.

Each node (computer connected to the Bitcoin network using a client that performs the task of validating and relaying transactions) gets a copy of the blockchain, which gets downloaded automatically upon joining the Bitcoin network." "...The full copy of the blockchain has records of every Bitcoin transaction ever executed. It can thus provide insight about facts, like how much value belonged a particular address at any point in the past."1 $A$ blockchain is like a financial ledger. In accounting, financial ledgers are done in INK. This is because you cannot change an entry, nor can you erase it. To correct a ledger entry, you must enter another entry to correct even a minor mistake in addition. There is a plus and minus side to a ledger and if your addition is incorrect and too high (debit) you must place an entry on the other side (credit) to remove or correct the initial debit, showing justification as to why the value changed. 
"Simply put, blockchain is a distributed ledger that provides a way for information to be recorded and shared by a community. In this community, each member maintains their own copy of the information, and all members must validate any updates collectively. The information could represent transactions, contracts, assets, identities, or practically anything else that can be described in digital form. Entries are permanent, transparent, and searchable, which makes it possible for community members to view transaction histories. Each update is a new "block" added to the end of the "chain." A protocol manages how new edits or entries are initiated, validated, recorded, and distributed. Crucially, privacy can also be selectively enforced, allowing varying degrees of anonymity or protection of sensitive information beyond those who have explicitly been given access. With blockchain, cryptology replaces third-party intermediaries as the keeper of trust, with all blockchain participants running complex algorithms to certify the integrity of the whole." ${ }^{2}$ Like a ledger every entry is permanent, even if in error, and is retained along with the correction in the block or chain depending on when corrected. Blockchain is designed to maintain this chain of transactions, or data indefinitely, securely, and completely while still allowing anonymity publically.

\section{The Unseen and Little Known Rules of Medical Records Systems}

Electronic medical records also have rules that mimic those of a financial ledger. Every entry automatically records the date and time entered, as well as the name of the user that entered the data. If that data is changed, the original entry is permanently archived in the database. If a doctor writes a report, then a day later modifies or adds to that report the initial report is archived and the current report is displayed. But inside the medical record system the doctor or an auditor can recall every copy of the report, even if it was modified 25 times. The history of the evolution of that report is available for anyone with access to the EMR system to review. So, you can't make a change without a trail, a reason, and proof that the person qualified actually made the change. It is just like a financial ledger in INK, except it also tells everyone exactly who changed what and exactly when they changed it.

One of the things Blockchain adds to this is transparency. Electronic medical record (EMR) systems do not share the entire history of an entry including changes. A 
blockchain based system must include every action or modification in linier order and is transparent. Doctors may push back against this until they admit they are human and make mistakes too. But for us patients, it forces everything to be visible. After all the record is not just about you, it is your property; and you deserve the whole truth, not the version best presented by the physician showing them as infallible. Besides, most corrections come from new findings and the discovery of these findings are trends that if transparent to all can offer new insights to diagnosis and treatment as early as the research phase.

\section{Blockchain Becomes the Solution for Globally Sharing Patient Data}

In a previous article, I explained that medical data is soon to be equal to 152 million years of $8 \mathrm{~mm}$ video, and that with or without standards that data are still distributed among 205,000 locations, making accurate collection of greater than $20 \%$ of that data impossible today, and at best only partly successful in the future. ${ }^{3}$ Even if totally standardized the distribution, distance, and collection methods would still be ineffective and prone to error.

Until now I had considered consolidation of data in central repositories like Digital Imaging and Communications in Medicine (DICOM) wants to as the solution-a standard for storing and transmitting medical images enabling the integration of medical imaging devices such as scanners, servers, and workstations. But I have faced unending resistance based on much business, legal, and patient privacy issues. The concept envisioned using blockchain actually removes the need for the current method of interoperability sharing, as well as the need for central collection of data to streamline access at every location.

You see, the concept of blockchain expects every system to be an equal data source. Because the data are a continuous chain of blocks of data, every system is part of the chain. On the internet, this transport, like TCP/IP (Transmission Control Protocol/Internet Protocol) is dependent on many possible sources for any given piece of data. To accomplish this smoothly, consistently, and accurately the most effective method is for every database to constantly keep every other database current. This 
means your in-house database has the same data for your patient as any other database in existence that has a record for the same patient.

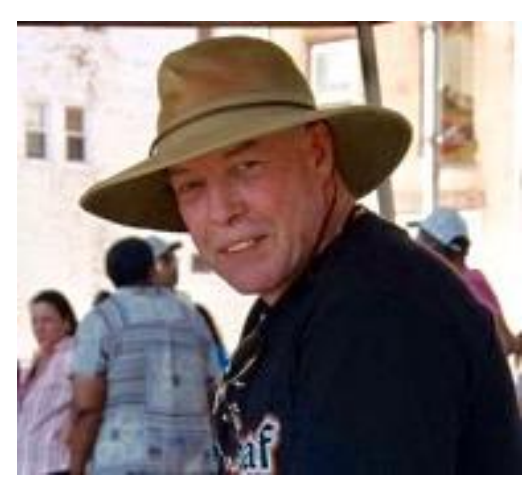

Matthew (Ted) Braid is an IT consultant and network engineer with thirty years' experience in the field of health care technology. He has lived in Baltimore, MD where he has worked at Johns Hopkins and many other progressive medical centers. As someone involved in the evolution of this field from its onset he has developed a passion for promoting the concept that medical technology must become a "Patient First" model for development, and that vendors need to accept the tradeoff of doing what is best for the patient and medicine as a whole, with the need for profit based on being unique and instead simply being better than the competition.

\section{References}

1. Anonymous. Block. Investopedia. URL: http://www.investopedia.com/terms/b/blockchain.asp\#ixzz4qt5tkAP2

2. Iansiti M, Lakhani KR. The truth about blockchain. Harvard Business Review. 2017. URL: https://hbr.org/2017/01/the-truth-about-blockchain

3. Braid, M. A plea for critical Changes to the Electronic Medical Record. Telehealth and Medicine today. 2017. URL: http://www.telhealthandmedtoday.com/a-pleafor-critical-changes-to-the-electronic-medical-record/ 
This is an open access article distributed in accordance with the Creative Commons Attribution Non-Commercial (CC BY-NC 4.0) license, which permits others to distribute, adapt, enhance this work non-commercially, and license their derivative works on different terms, provided the original work is properly cited and the use is noncommercial. See: http://creativecommons.org/licenses/by-nc/4.0.

Category: Opinion and Interview

Tags: electronic medical record, EMR, improved patient care, patient, standardization of telehealth, financial tracking in telehealth, electronic health record environment 